simulators may help formal laparoscopic training to overcome the lack of exposure to laparoscopic procedures as primary surgeons.

Dr. Fernando J. Kim

Chief of Urology, Denver Health Med. Ctr. Associate Professor, Univ. Colorado Health Sci. Ctr. Director of Minimally Invasive Urol. Oncology, UCHSC

Denver, Colorado, USA

E-mail:fernando.kim@dhha.org

\title{
IMAGING
}

\section{T1 hyperintense renal lesions: characterization with diffusion-weighted MR imaging versus contrast-enhanced MR imaging}

Kim S, Jain M, Harris AB, Lee VS, Babb JS, Sigmund EE, Rueff LE, Taouli B

Department of Radiology, New York University Medical Center, New York, NY, USA

Radiology. 2009; 251: 796-807

Purpose: To compare the performance of apparent diffusion coefficient (ADC) measurement obtained with diffusion-weighted (DW) magnetic resonance (MR) imaging in the characterization of non-fat-containing T1 hyperintense renal lesions with that of contrast material-enhanced MR imaging, with histopathologic analysis and follow-up imaging as the reference standards.

Materials and Methods: Institutional review board approval was obtained for this HIPAA-compliant retrospective study, and the informed consent requirement was waived. Two independent observers retrospectively assessed MR images obtained in 41 patients with non-fat-containing T1 hyperintense renal lesions. The MR examination included acquisition of DW and contrast-enhanced T1-weighted images. For each index lesion, the observers assessed the (a) mean (+/- standard deviation) of ADC, (b) enhancement ratio, and (c) subtracted images for the presence of enhancement (confidence score, 1-5). Histopathologic analysis of renal cell carcinomas (RCCs) and follow-up imaging for benign lesions were the reference standards. ADCs of benign lesions and RCCs were compared. Receiver operating characteristic (ROC) curve analysis was performed to assess the accuracy of DW imaging, enhancement ratio, and subtraction for the diagnosis of RCC.

Results: A total of 64 lesions (mean diameter, $3.9 \mathrm{~cm}$ ), including 38 benign T1 hyperintense cysts and $26 \mathrm{RCCs}$, were assessed. Mean ADCs of RCCs were significantly lower than those of benign cysts $([1.75+/-0.57] \mathrm{x}$ $10(-3) \mathrm{mm}(2) / \mathrm{sec}$ vs $[2.50+/-0.53]$ x $10(-3) \mathrm{mm}(2) / \mathrm{sec}, \mathrm{P}<.0001)$. ADCs of solid and cystic portions of complex cystic RCCs were significantly different $([1.37+/-0.55]$ x $10(-3) \mathrm{mm}(2) / \mathrm{sec}$ vs $[2.45+/-0.63]$ x $10(-3)$ $\mathrm{mm}(2) / \mathrm{sec}, \mathrm{P}<.0001)$. When data from both observers were pooled, area under the ROC curve, sensitivity, and specificity were $0.846,71 \%$, and $91 \%$, respectively, for DW imaging; $0.865,65 \%$, and $96 \%$, respectively, for enhancement ratio (at the excretory phase); and $0.861,83 \%$, and $89 \%$, respectively, for subtraction $(\mathrm{P}=.48$ and $\mathrm{P}=.85$, respectively). The combination of DW imaging and subtraction resulted in area under the ROC curve, sensitivity, and specificity of $0.893,87 \%$, and $92 \%$, respectively, with significantly improved reader confidence compared with subtraction alone $(\mathrm{P}=.041)$.

Conclusion: The performance of DW imaging was equivalent to that of enhancement ratio in the characterization of T1 hyperintense renal lesions, with both methods having lower sensitivity than image subtraction without reaching significance. 


\section{Editorial Comment}

On MR imaging, most renal masses are hypointense on T1 and hyperintense on T2, thus comparison between T1-weighted image pre and post intravenous injection of contrast readily shows variable degree of hyperintensity of the lesions due to contrast enhancement (particularly hypervascular ones). Some lesions, however, are hyperintense in T1-weighted images making the perception of contrast enhancement a very difficult task. These hyperintense lesions on T1-weighted images are either benign (hemorrhagic cyst, hematoma, vascular lesion or oncocitoma) or malignant masses (papillary renal cell carcinoma). Image subtraction technique is very useful for the demonstration of subtle contrast enhancement in hyperintense T1 lesions. Image subtraction however can be of limited value in patients with irregular respiratory movements, which precludes adequate images subtraction.

DW imaging has been used to assess several renal disorders: infection, ischemia, obstruction and masses. The authors of this manuscript show that DW imaging can be of value to characterize non-fat-containing T1 hyperintense lesions. They found that the diffusion is more restricted in renal cell carcinoma (lower ADC values) than in benign hemorrhagic or proteinaceous cyst. Although with lower sensitivity than that image subtraction, the authors recommend DW imaging as an alternative to contrast-enhanced MRI in patients with chronic renal insufficiency that are at risk for development of nephrogenic system fibrosis secondary or associated with gadolinium-containing agent.

Dr. Adilson Prando

Chief, Department of Radiology and Diagnostic Imaging, Vera Cruz Hospital

Campinas, São Paulo, Brazil

E-mail: adilson.prando@gmail.com

\section{Imaging appearance of granulomatous disease after intravesical Bacille Calmette-Guerin (BCG) treatment of bladder carcinoma}

Ma W, Kang SK, Hricak H, Gerst SR, Zhang J

Department of Radiology, Memorial Sloan-Kettering Cancer Center, New York, NY, USA

AJR Am J Roentgenol. 2009; 192: 1494-500

Objective: The purpose of our study is to present the radiographic findings in a series of 16 patients with complications associated with intravesical bacille Calmette-Guérin (BCG) treatment of bladder cancer.

Conclusion: Intravesical BCG-related complications such as granulomatous disease may show imaging findings mimicking primary or metastatic tumors in patients with bladder cancer. Radiologists should consider this possibility when imaging abnormalities are encountered in bladder cancer patients treated with intravesical BCG so that appropriate management can be administered and unnecessary procedures avoided.

\section{Editorial Comment}

Intravesical therapy with bacillus Calmette-Guérin (BCG) has proved to be more effective in the prophylaxis and treatment of superficial bladder tumors and carcinoma in situ than most chemotherapeutic agents. Some complications however may occur with this treatment. Granulomatous reaction may occur either in the urinary tract or sporadically outside the urinary tract (hepatitis, disseminated infection, miliary tuberculosis and polyarthritis).

This manuscript calls the attention of radiologists and urologists regarding the imaging manifestations of these complications. As it shown by the authors, granulomatous reaction may resemble focal primary renal or bladder tumors and focal or diffuse prostatic tumors. Since instillation of BCG is indicated for treatment of 